\title{
Knockdown of serine/threonine protein phosphatase 5 enhances gemcitabine sensitivity by promoting apoptosis in pancreatic cancer cells in vitro
}

\author{
JINHUI ZHU ${ }^{1}$, YUN JI $^{2}$, YUANQUAN YU ${ }^{2}$, YUN JIN $^{2}$, XIAOXIAO ZHANG ${ }^{2}$, \\ JIALE ZHOU $^{2}$ and YAN CHEN ${ }^{1}$
}

Departments of ${ }^{1}$ General Surgery and Laparoscopic Center, and ${ }^{2}$ General Surgery, Second Affiliated

Hospital of Zhejiang University School of Medicine, Hangzhou, Zhejiang 310009, P.R. China

Received July 13, 2016; Accepted September 22, 2017

DOI: $10.3892 / \mathrm{ol} .2018 .8363$

\begin{abstract}
The targeting protein of serine/threonine protein phosphatase 5 (PPP5C) has been reported to be present in various malignancies. However, its functional role in pancreatic cancer (PC) remains unknown. In the present study, the function of PPP5C in PC cells treated with the first-line drug gemcitabine (GEM) was investigated. Short hairpin (sh)RNA targeting PPP5C was constructed to knockdown PPP5C in PANC-1 cells. Cell cycle and apoptosis analyses were performed in order to investigate the mechanisms underlying the effects induced by PPP5C silencing combined with GEM treatment. Western blot analysis was applied to detect the expression of certain key regulators of cell apoptosis in PANC-1 cells treated with GEM. shRNA against PPP5C effectively suppressed the proliferation of PANC-1 cells treated with GEM. Additionally, cell cycle analysis indicated that PPP5C knockdown resulted in a higher number of PANC- 1 cells treated with GEM in $\mathrm{G}_{0} / \mathrm{G}_{1}$ phase arrest. Knockdown of PPP5C increased the expression of associated apoptotic markers, including cleaved caspase 3, poly (ADP-ribose) polymerase and phosphorylated (p)-p53. In addition, the combination of treatment with GEM and PPP5C silencing significantly increased the apoptosis of PANC-1 cells by affecting the expression levels of p-c-Jun $\mathrm{N}$-terminal kinases and p-p38. The present study suggests that PPP5C may be a potential target for the treatment of $\mathrm{PC}$ and that it may enhance the gemcitabine sensitivity of PC cells.
\end{abstract}

Correspondence to: Dr Yan Chen, Department of General Surgery and Laparoscopic Center, Second Affiliated Hospital of Zhejiang University School of Medicine, 88 Jiefang Road, Hangzhou, Zhejiang 310009, P.R. China

E-mail: chenyan1256@126.com

Key words: protein phosphatase 5 catalytic subunit, pancreatic cancer, gemcitabine, cell proliferation, apoptosis

\section{Introduction}

Pancreatic cancer (PC) is one of the most lethal malignancies of the human digestive system due to its rapid progression, high recurrence rate and strong chemoresistance $(1,2)$. PC is rarely diagnosed at an early stage due to the fact that patients with localized PC have no recognizable signs or symptoms. Therefore, the majority of PC patients do not receive a definitive diagnosis until late, once the PC cells have metastasized to other organs $(3,4)$. Even in cases where surgical resection is performed, the local recurrence rate of $\mathrm{PC}$ is high and the 5-year survival rate remains at only $5 \%$ following surgery. Despite recent progress in chemotherapeutics and understanding of the molecular biological mechanisms of PC, limited progress has been made in therapeutic methods for metastatic disease. Due to the fact that the incidence of PC has been markedly increasing over recent years (5), it is necessary to find novel therapies for PC (6).

Gemcitabine (2',2'-difluoro-2'-deoxycytidine, dFdC; GEM), a pyrimidine analog, which is phosphorylated to diphosphate and triphosphate forms to inhibit DNA polymerase and ribonucleotide reductase (7), is now used as the standard palliative treatment for PC (8-11). Originally, GEM was approved as the first-line treatment for PC by the US Food and Drug Administration in 1997 based upon the study by Burris et al (9). Almost 2 decades later, GEM was approved for the treatment of advanced PC. GEM has demonstrated marked effects on the survival time of PC patients when used in various forms of therapy, including GEM monotherapy, combination treatment with GEM and in a number of other active cytotoxic agents or regimens (12-15). However, there are a number of factors that have been reported to cause GEM resistance (16).

The reversible phosphorylation of proteins serves a crucial function in regulating numerous biological responses. In mammalian cells, $>99 \%$ of this phosphorylation occurs on serine or threonine (Ser/Thr) residues. This type of protein phosphatase dephosphorylates a range of proteins involved in a wide range of cellular processes, including apoptosis, cell differentiation, cell survival, the response to DNA damage, and the regulation of ion channels and 
circadian rhythms $(17,18)$. The reversible phosphorylation of proteins may also impact on several signaling pathways, including those controlled by kinases, such as apoptosis signal-regulating kinase $1 /$ mitogen-activated protein kinase kinase kinase 5 (ASK1/MAP3K5), protein kinase, DNA-activated, catalytic polypeptide and RAF1 (19-21). The targeting protein of Ser/Thr protein phosphatase 5 (PPP5C), a member of the phosphoprotein phosphatase (PPP) family of $\mathrm{Ser} / \mathrm{Thr}$ phosphatases, is an enzyme encoded by the PPP5C gene (22). PPP5C is broadly expressed and has distinct structural properties compared with other phosphatases in the PPP family $(23,24)$. PPP5C belongs to the protein phosphatase- 5 subfamily and contains only 1 single polypeptide chain (25-27). It has been reported that PPP5 functions upstream of p53 and that it phosphorylates p53 to regulate the induction of p21 (WAF1/Cip1), as well as to mediate the growth arrest pathway $(25,28)$. PPP5C has been demonstrated to interact with ASK1 (19), cryptochrome circadian clock 2 (29), guanine nucleotide-binding protein subunit $\alpha-12$ (30) and Ras-related C3 botulinum toxin substrate 1 (31). ASK1 could activate c-Jun N-terminal kinase (JNK) and p38 mitogen-activated protein kinases (MAPK) in a Raf-independent manner in response to a number of stresses. ASK1 is additionally associated with cancer, diabetes, and cardiovascular and neurodegenerative diseases (32).

The result of PPP5C-knockdown by siRNA or oligonucleotides has revealed that PPP5C is also associated with the stress response and inhibition of the proliferation rate of tumor cells, including ovarian cancer (33), glioma (34) and liver carcinoma (35) cells. Additionally, a previous study demonstrated that an elevated level of PPP5C protein is directly associated with Alzheimer's disease $(35,36)$. However, the function of PPP5C in PC has not been reported prior to the present study. In the present study, shRNA was constructed to silence the expression of PPP5C in the PC PANC-1 cell line and the effects of PPP5C on PANC-1 cells treated with GEM were also investigated.

\section{Materials and methods}

Cell lines and cell culture. The human embryonic kidney 293 cell line (HEK 293) and the human PC cell line PANC-1 were used in the present study and were purchased from the Cell Bank of the Chinese Academy of Sciences (Shanghai, China). The cell lines were cultured in Dulbecco's modified Eagle's medium (Hyclone; GE Healthcare Life Sciences, Logan, UT, USA; cat. no. SH30243.01B+), supplemented with $10 \%$ fetal bovine serum (Biological Industries, Beit-Haemek, Israel; cat. no. 04-001-1A) and maintained at $37^{\circ} \mathrm{C}$ in a humidified incubator with $5 \% \mathrm{CO}_{2}$.

Construction of the lentivirus vector for PPP5C short hairpin (sh)RNA and virus packaging. Based upon the sequence of PPP5C (NM_001204284.1), a responsible shRNA sequence (5'-GAGACAGAGAAGATTACAGTACTCGAGTACTGTAAT CTTCTCTGTCTCTTTTT-3') was generated to target PPP5C and a control shRNA sequence (5'-TTCTCCGAACGTGTCACG TCTCGAGACGTGACACGTTCGGAGAA-3') was designed and synthesized. The recombinant vectors, PPP5C shRNA (shPPP5C) and control shRNA (shCon), were designated to carry the corresponding shRNA. T4 DNA ligase (New England BioLabs, Inc., Ipswich, MA, USA) was used to construct shRNA fragments (50 ng), which were cloned into the lentiviral expression vector pGP (Shanghai Hollybio, Shanghai, China) prior to being digested by EcoRI and BamHI (New England BioLabs, Inc.). Lentivirus was generated by co-transfection of HEK293 cells with recombinant vectors and packaging plasmids (pVSVG-I and pCMV $\Delta$ R8.92; Shanghai Hollybio). The supernatants were collected $96 \mathrm{~h}$ after transfection to extract the lentivirus that may express PPP5C shRNA or control shRNA. The lentivirus was then purified via ultracentrifugation in a condition of $100,000 \mathrm{xg}$ for $30 \mathrm{~min}$ at $4^{\circ} \mathrm{C}$. PANC-1 cells were infected with the concentrated virus at a multiplicity of infection of 10 and mock-infected cells were used as negative controls. Since the lentivirus carries a green fluorescence protein (GFP) as a reporter and this GFP is driven by the cytomegalovirus promoter, the titer of lentivirus was determined by counting the number of cells that expressed GFP under fluorescence microscopy under x100 magnification (Olympus Corporation, Tokyo, Japan) following $48 \mathrm{~h}$ of infection. The efficiency of PPP5C-knockdown was subsequently measured by reverse transcription quantitative polymerase chain reaction (RT-qPCR) and western blot analysis.

RNA extraction and $R T-q P C R$. PANC-1 cells were harvested following $72 \mathrm{~h}$ of lentivirus infection and all of the RNA from the cultured cells was extracted using TRIzol reagent (Invitrogen; Thermo Fisher Scientific, Inc., Waltham, MA, USA; cat. no. 15596-026) according to the manufacturer's protocols. The purity and integrity of extracted RNA was assessed using spectrophotometry and agarose gel electrophoresis, respectively. The first strand in the complementary DNA of the extracted RNA was synthesized from the aforementioned RNA $(2 \mu \mathrm{g})$ using reverse transcription reagents (Promega Corporation, Madison, WI, USA; cat. no. M1705). The primers used in this study were: PPP5C forward, 5'-CCCAACTACTGCGACCAG AT-3' and reverse, 5'-CCCGTCACCTCACATCATTC-3'; and $\beta$-actin forward, 5'-GTGGACATCCGCAAAGAC-3' and reverse, 5'-AAAGGGTGTAACGCAACTA-3'. The expression of PPP5C mRNA was evaluated by RT-qPCR using the SYBR Green Core Reagents kit on BioRad CFX96 Touch $^{\mathrm{TM}}$ Real-Time PCR system (Bio-Rad Laboratories, Inc., Hercules, CA, USA). The conditions of the PCR involved incubating all of the samples at $95^{\circ} \mathrm{C}$ for $1 \mathrm{~min}$, followed by 40 cycles of denaturation at $95^{\circ} \mathrm{C}$ for $5 \mathrm{sec}$, and annealing and extension at $60^{\circ} \mathrm{C}$ for $20 \mathrm{sec}$. $\beta$-actin was used as the input reference. The relative gene expression levels were quantified using the $2^{-\Delta \Delta \mathrm{Cq}}$ method (37).

Western blot analysis. PANC-1 cells were harvested following 6 days of lentivirus infection. Cells were harvested and washed twice using ice-cold phosphate-buffered saline (PBS) and then lysed in ice-cold 2X SDS Lysis Buffer [100 mM Tris-HCl (pH 6.8), $10 \mathrm{mM}$ EDTA, 4\% SDS and $10 \%$ glycine]. The protein concentration of cell lysate was determined using the BCA protein assay kit (Pierce; Thermo Fisher Scientific, Inc.; cat. no. 23235). Extracted proteins $(30 \mu \mathrm{g})$ were separated on 10\% SDS-PAGE prior to being transferred electrophoretically onto a polyvinylidene fluoride membrane (Bio-Rad Laboratories, Inc.; cat. no. 162-0177). 
The proteins were blocked in $5 \%$ skimmed milk for $1 \mathrm{~min}$ at room temperature and probed with specific antibodies at $4^{\circ} \mathrm{C}$ overnight. The primary antibodies used were rabbit anti-PPP5C (Proteintech Group Inc., Chicago, IL, USA; cat. no. 117515-1-AP; 1:1,000 dilution), and rabbit anti-p-JNK (cat. no. 4668, 1:1,000 dilution), rabbit anti-JNK (cat. no. 9252; 1:1,000 dilution), rabbit anti-p-p38 (cat. no. 9215; 1:500 dilution), rabbit anti-p38 (cat no. 9212; 1:1,000 dilution), rabbit anti-caspase3 (cat. no. 9661; 1:500 dilution), rabbit anti-PARP (cat. no. 9542; 1:1,000 dilution), rabbit anti-p-p53(Ser315) (cat. no. 2528; 1:500 dilution) and rabbit anti-p53 antibody (Ser15) (cat. no. 11094; 1:500 dilution) (all from Cell Signaling Technology, Inc., Danvers, MA, USA). One rabbit anti-GADPH antibody was used as loading control (cat. no. 11205; 1:1,000 dilution; Proteintech Group Inc.). Subsequently, the membranes were washed 3 times in Tris-buffered saline (TBST) prior to being incubated with goat anti-rabbit Immunoglobulin $\mathrm{G}$ horseradish peroxidase-conjugated secondary antibody (cat. no. SC-2054; 1:5,000 dilution; Santa Cruz Biotechnology, Inc., Dallas, TX, USA) for $1 \mathrm{~h}$ at room temperature. In addition, the JNK blot was stripped and reprobed with p-JNK, p38 was stripped and reprobed with $\mathrm{p}-38$, and PARP was stripped and reprobed with p-38. Stripping buffers consisted of $0.94 \mathrm{~g}$ glycine (cat. no. GB0235; Sangon Biotech Co., Ltd., Shanghai, China) and 5 g sodium dodecyl sulfonate (cat. no. A500228; Sangon Biotech Co., Ltd.) in $500 \mathrm{ml}$ water. The blot was placed into the stripping buffers prior to being agitated in the shaker for $8 \mathrm{~min}$ at room temperature. Subsequently, the blot was washed in 1X TBST 3 times, for $5 \mathrm{~min}$ each time. The blot was then blocked and reacted with all antibodies indicated as above in the aforementioned manner. The target bands were visualized using an enhanced chemiluminescence kit (GE Healthcare Life Sciences, Uppsala, Sweden) according to the manufacturer's protocols.

MTT assay. Cell proliferation and viability were determined by MTT assay. Cells were plated at a density of $5 \times 10^{3}$ cells/well in 96-well culture plates following $72 \mathrm{~h}$ of lentivirus infection and treatment with various concentrations of GEM (1, 5 and $10 \mu \mathrm{M})$. Next, $20 \mu \mathrm{l}$ MTT solution ( $5 \mathrm{mg} / \mathrm{ml}$ dissolved in PBS) was added to each well followed by $4 \mathrm{~h}$ of incubation at $37^{\circ} \mathrm{C}$. Following incubation, $100 \mu \mathrm{l}$ stop buffer acidic isopropanol (0.01 M HCl, 10\% SDS and 5\% isopropanol) was added to each well to stop the reaction. The culture plates with incubated cells were gently agitated for 10 min and analyzed using an Epoch Microplate Spectrophotometer version 2 (BioTek Instruments, Inc., Winooski, VT, USA) at a wavelength of $595 \mathrm{~nm}$.

Cell cycle analysis. Cell cycle analysis was conducted using propidium iodide (PI) according to the manufacturer's protocols. PANC-1 cells were seeded onto $6-\mathrm{cm}$ wide dishes at a density of $5 \times 10^{5}$ cells/well. Following lentivirus infection for 4 days and treatment with $5 \mu \mathrm{M}$ GEM, PANC-1 cells were collected, washed, fixed with $75 \%$ ethanol at $4^{\circ} \mathrm{C}$ overnight and then stained with PI. Finally, the distribution of the cell cycle was assessed using a fluorescence-associated cell sorting (FACS) assay with FACSCalibur (Beckman Coulter, Inc., Brea, CA, USA) and the results were analyzed using ModFit LT 3.1 software (Verity Software House, Inc., Topsham, ME, USA).

Annexin $V$ staining apoptosis analysis. To assess the rate of apoptosis, PANC-1 cells infected with lentivirus and treated with $1 \mu \mathrm{M}$ GEM were stained using the Annexin V-APC/7-AAD Apoptosis Detection kit (cat. no. KGA1026; Nanjing KeyGen Biotech Co., Ltd., Nanjing, China). The cells were analyzed on a FACSCalibur (Beckman Coulter, Inc.) using the CellQuest Pro software (version 5.1) (BD Biosciences, San Jose, CA, USA) and the percentage of each quadrant was calculated using this software.

Statistical analysis. All results are expressed as the mean \pm standard deviation. Statistical analysis was performed using unpaired Student's t-test or one-way analysis of variance followed by Dunnett's multiple comparisons test. $\mathrm{P}<0.05$ was considered to indicate a statistically significant difference. Statistical analysis was performed using SPSS 13.0 statistical software (SPSS Inc., Chicago, IL, USA).

\section{Results}

Endogenic PPP5C is inhibited by shPPP5C and induced by GEM in the PC PANC-1 cell line. To investigate the role served by PPP5C in PC, the PANC-1 cell line was infected with a lentivirus vector carrying shRNA targeting PPP5C. PPP5C-knockdown efficiency was verified using RT-qPCR and western blot analysis. Compared with that in the negative control cells (shCon), the PPP5C mRNA expression in PPP5C shRNA-infected PANC-1 cells (shPPP5C) was decreased by $94 \%$ (Fig. 1A). Likewise, PPP5C protein expression was significantly decreased in the PANC-1 cells infected with shPPP5C (Fig. 1B). In addition, the effect of GEM on PPP5C expression in the PANC-1 cells was detected by qRT-PCR and western blot analysis. The results indicated that GEM could increase intracellular PPP5C mRNA and protein expression at concentrations of 1,5 and $10 \mu \mathrm{m}$ compared with the untreated control (Fig. 1C and D). These results indicate that intracellular PPP5C was successfully silenced and that it could be enhanced by GEM in PANC-1 cells.

PPP5C silencing enhances the chemosensitivity of PANC-1 cells to GEM. To determine whether or not PANC-1 cells with reduced PPP5C expression were more sensitive to GEM, PANC-1 cells were stably transfected with shPPP5C or shCon using a continuous 72-h MTT assay. As demonstrated in Fig. 2A, shPPP5C markedly inhibited the proliferation of PANC-1 cells compared with shCon. Following addition of GEM at varying concentrations, the cell growth rates were significantly decreased (Fig. 2B-D) compared with shCon cells (Fig. 2A) using a Student's t-test. As demonstrated in Fig. 2E, the inhibition ratio was significantly increased in the shPPP5C group following GEM treatment. In addition, statistical analysis revealed that regardless of whether or not PPP5C was silenced, the inhibitory effect of 5 or $10 \mu \mathrm{M}$ GEM exhibited no marked differences at 24, 48 and $72 \mathrm{~h}$, indicating that the effect of GEM had reached saturation (Fig. 2E). Even if the effect of GEM had reached saturation, the shPPP5C 
A

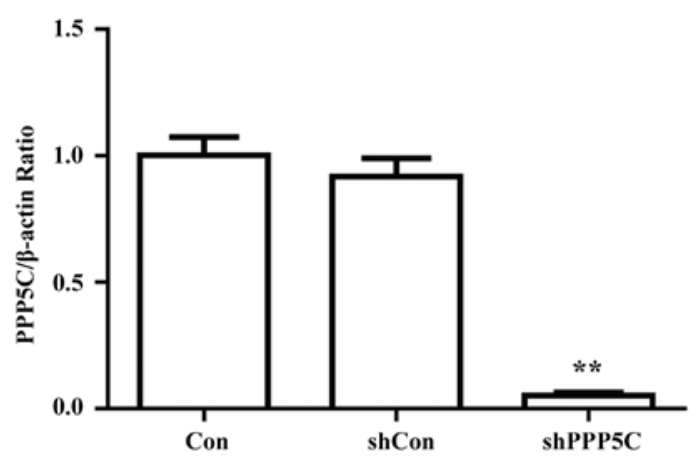

C

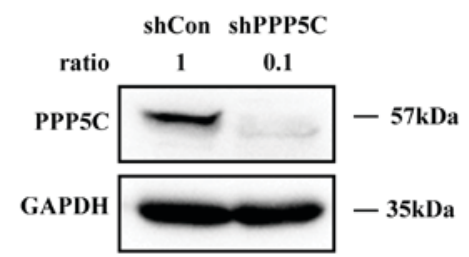

B

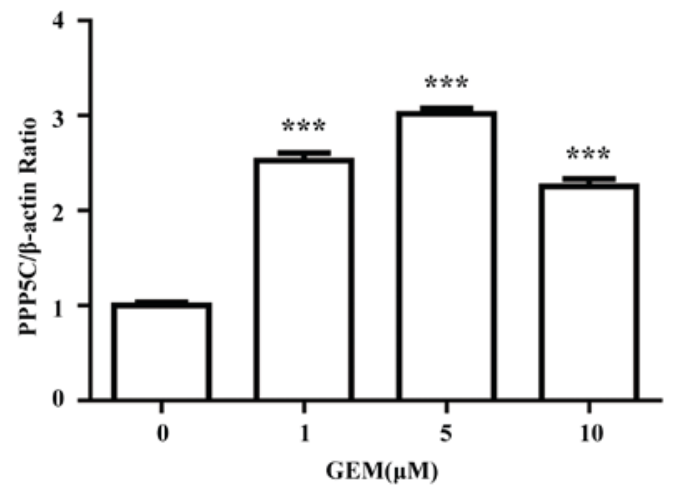

D

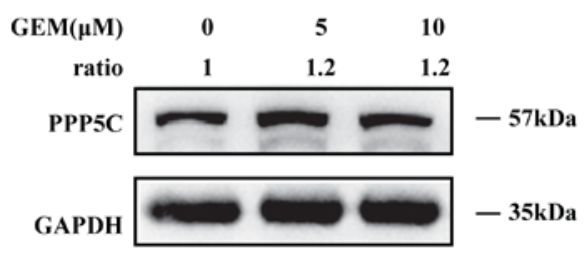

Figure 1. Expression of PPP5C in the PC PANC-1 cell line. (A) Analysis of PPP5C expression in PANC-1 cells transduced with shCon and shPPP5C by RT-qPCR analysis. (B) Analysis of PPP5C expression in PANC-1 cells transduced treated with GEM at different concentrations by RT-qPCR analysis. (C) Analysis of PPP5C expression in PANC-1 cells transduced with shCon and shPPP5C by western blot analysis. (D) Analysis of PPP5C expression in PANC-1 cells transduced treated with GEM at different concentration by western blot analysis. Data are presented as the mean \pm standard deviation of 3 independent experiments. Statistical analysis in A and B were performed using one-way ANOVA followed by Dunnett's multiple comparisons test. ${ }^{* *} \mathrm{P}<0.01$ vs. control, ${ }^{* * *} \mathrm{P}<0.001$ vs. $0 \mu \mathrm{g}$ GEM. PPP5C, serine/threonine protein phosphatase 5; shCon, short hairpin control; shPPP5C, short hairpin PPP5C; RT-qPCR, reverse transcription-quantitative polymerase chain reaction; GEM, gemcitabine; ANOVA, analysis of variance.

group remained able to further inhibit PANC-1 cell proliferation compared with the shCon group (the inhibition ratio at different GEM concentrations exhibited no dose-response effect; Fig. 2E). These results indicate that PPP5C-knockdown sensitizes PANC-1 cells to GEM treatment.

GEM enhances shPPP5C-induced PANC-1 cell cycle arrest and apoptosis. In order to identify the mechanisms underlying the antiproliferation effect, the distribution of cells in the cell cycle phases was analyzed using flow cytometry (Fig. 3A). The proportion of G0/G1 phase cells was markedly increased while the $\mathrm{G} 2 / \mathrm{M}$ phase population was decreased under GEM treatment when compared with PANC-1 cells treated with the single shCon or shPPP5C alone (Fig. 3B and $\mathrm{C}$ ). These data suggested that the combined treatment could further arrest the cell cycle at the $\mathrm{G}_{0} / \mathrm{G}_{1}$ phase in PANC-1 cells. In addition, GEM treatment plus PPP5C silencing resulted in a significant increase in the cell population in the sub-G1 phase (Fig. 3D), suggesting the presence of cell apoptosis.

To further examine the effect of combined treatment and that of PPP5C silencing alone on cell apoptosis in PANC-1 cells, Annexin V-APC/7-AAD staining was performed. As demonstrated in (Fig. 3E and F), flow cytometry analysis revealed that the percentage of early apoptotic cells (Annexin $\mathrm{V}^{+} / 7-\mathrm{AAD}^{-}$) was higher in the shPPP5C group compared with that in the shCon group, while no difference was observed in the percentage of late apoptotic cells (Annexin $\mathrm{V}^{+} / 7-\mathrm{AAD}^{+}$) between the shPPP5C and shCon groups. The percentages of early and late apoptotic cells were revealed to be significantly increased in the shPPP5C group when PPP5C silencing was combined with GEM treatment in comparison with cells treated with shCon, shPPP5C or GEM alone. More specifically, the apoptosis rate (for early and late apoptotic cells) was $~ 35.08 \%$ in the group treated with GEM and shPPP5C, which was significantly higher than that in the shCon (15.57\%), shPPP5C (22.46\%) or GEM plus shCon (20.64\%) groups. These results further corroborated the hypothesis that silencing of PPP5C enhanced the apoptotic effect of GEM in vitro.

Mechanism of GEM-enhanced shPPP5C-induced PANC-1 cell apoptosis. To investigate the underlying mechanism of GEM-enhanced shPPP5C-induced PANC-1 cell apoptosis, apoptosis-associated proteins were determined by western blot analysis (Fig. 4). The results demonstrated that the protein expression of cleaved caspase 3 and poly (ADP-ribose) polymerase (PARP) was increased in the shPPP5C group compared with that in the shCon group (Fig. 4A and C). Additionally, the expression of tumor suppressor p53 was also determined and the protein expression ratio of $\mathrm{p}$-p53/p53 was also upregulated (Fig. 4B and C). In addition, the association between MAPK family-associated proteins (JNK, p-JNK, p38 and p-p38) and shPPP5C-mediated apoptosis was investigated. As demonstrated in (Fig. 4A and C), the protein expression ratio of $\mathrm{p}-\mathrm{JNK} / \mathrm{JNK}$ was increased, while almost no change was observed in that of $\mathrm{p}-\mathrm{p} 38 / \mathrm{p} 38$. These results indicate that the effect of silencing PPP5C on cell apoptosis resulted from the altered expression of the associated anti-apoptotic proteins. Additionally, the combined treatment of GEM and 
A

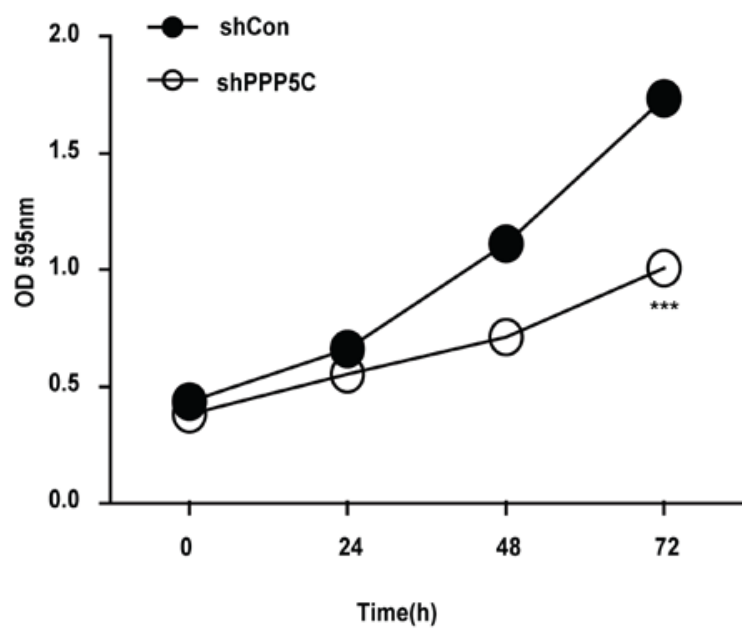

C

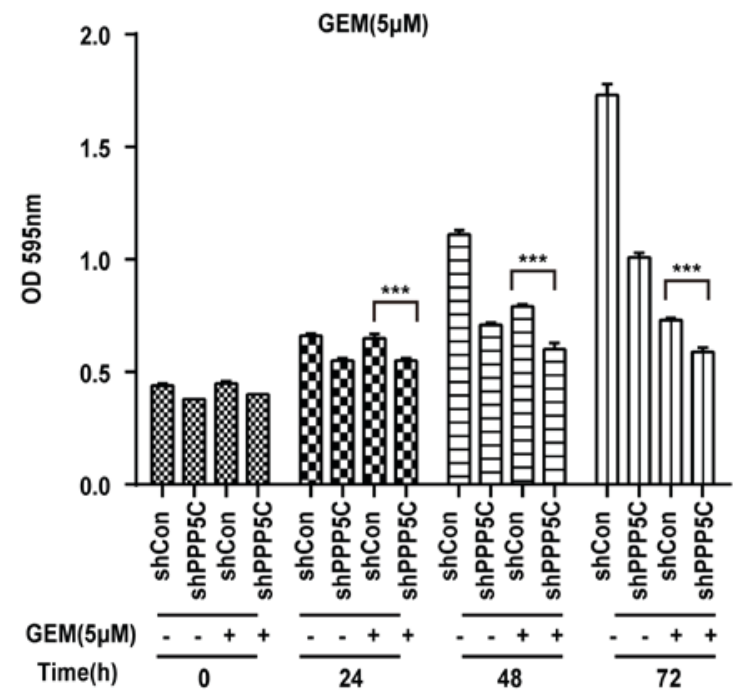

B

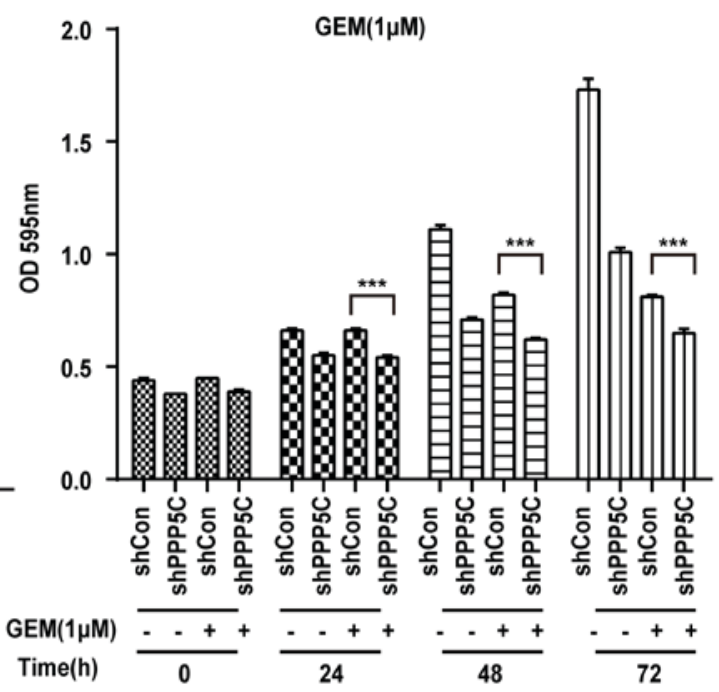

D

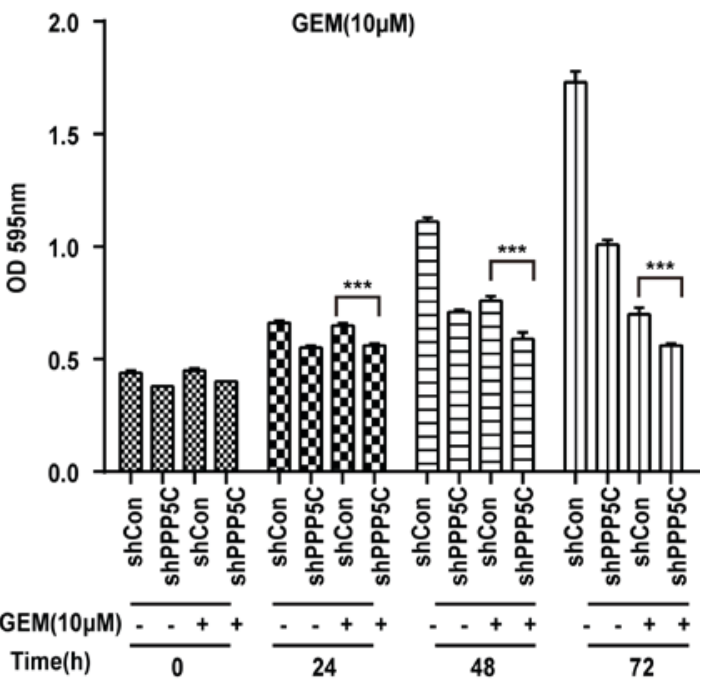

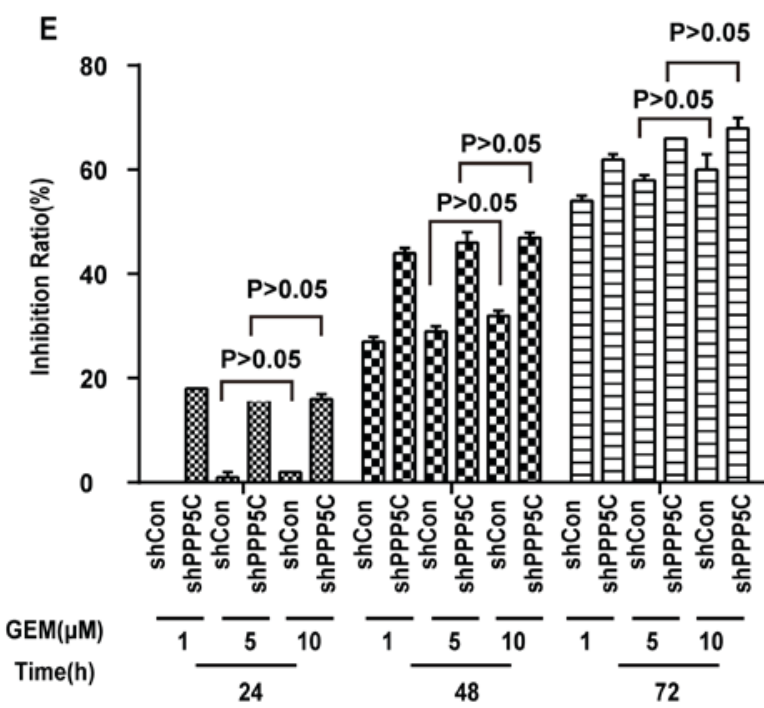

Figure 2. PPP5C silencing increases sensitivity to GEM in PANC-1 cells. (A) The proliferation ability of PANC-1 cells infected with shPPP5C was measured by MTT assay. (B) The cell growth inhibition rate following addition $1 \mu \mathrm{M}$ GEM were measured by MTT assay. (C) The cell growth curves following addition $5 \mu \mathrm{M}$ GEM were measured by MTT assay. (D) The cell growth curves following addition $10 \mu \mathrm{M}$ GEM were measured by MTT assay. (E) Cell growth inhibition rate. Data are presented as the mean \pm standard deviation of 3 independent experiments. ${ }^{* * * *} \mathrm{P}<0.001$. PPP5C, serine/threonine protein phosphatase 5; GEM, gemcitabine; shPPP5C, short hairpin PPP5C; shCon, short hairpin control; OD, optical density. 
A

shCon

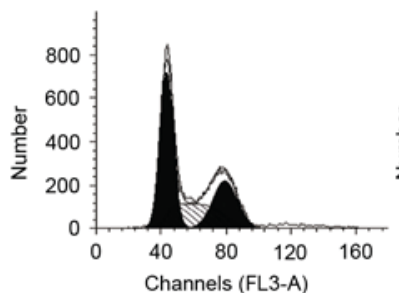

B
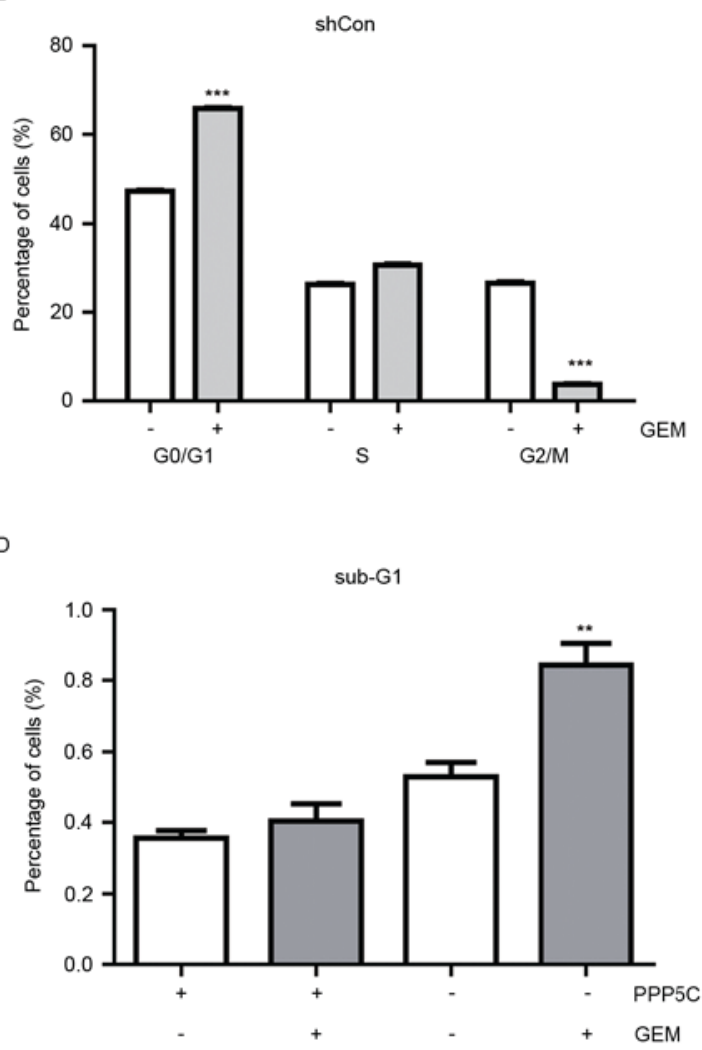
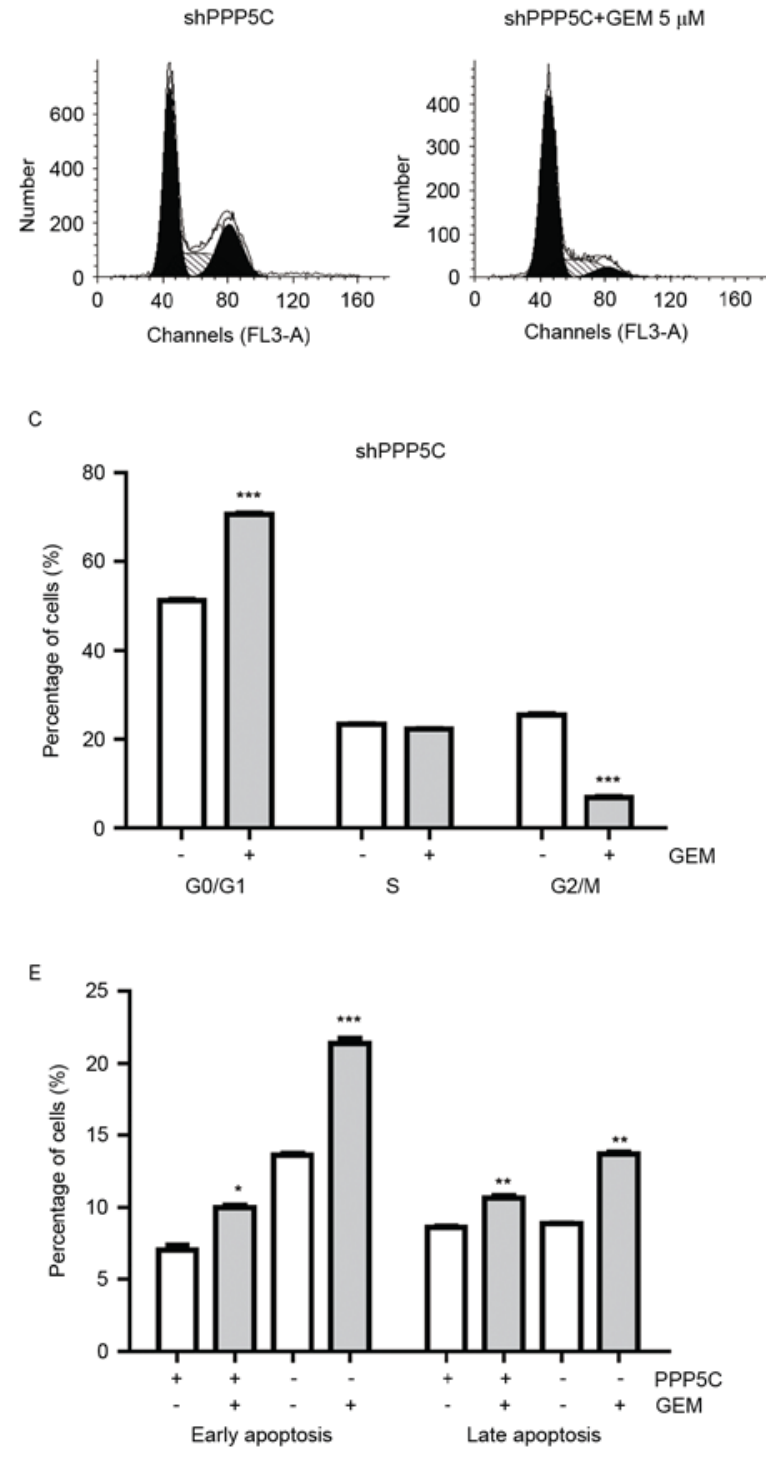

$\mathrm{F}$
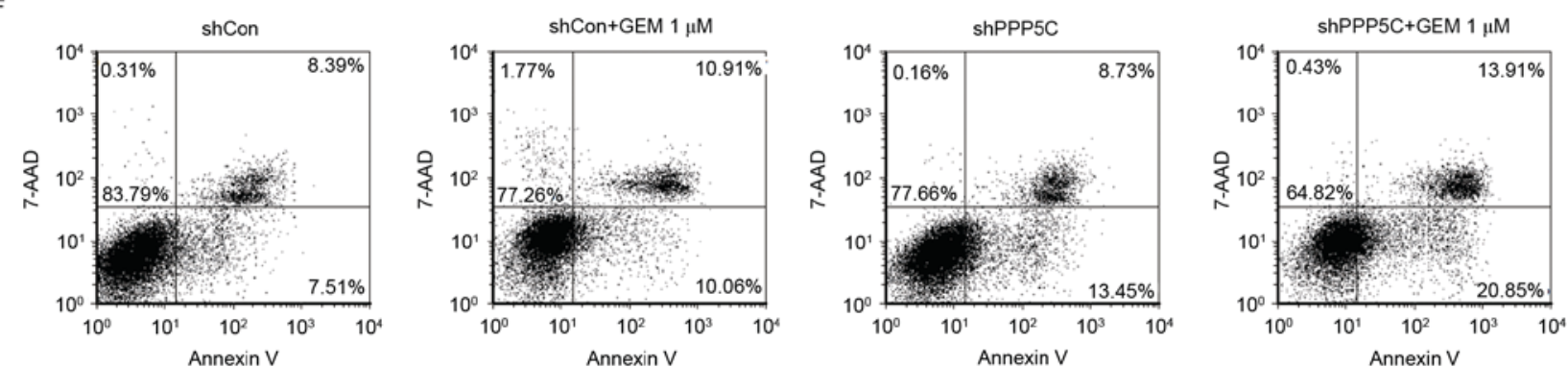

Figure 3. GEM enhances shPPP5C-induced PANC-1 cell cycle arrest and apoptosis. (A) Cell cycle distribution was analyzed by flow cytometry. Representative images of shCon, shPPP5C and the combined treatment of GEM and shCon or shPPP5C measured by fluorescence-activated cells sorting analyses. (B) Quantification and statistical analysis of the cell percentages in the different cell cycle phases in shCon group. (C) Quantification and statistical analysis of the cell percentages in the different cell cycle phases in shPPP5C group. (D) Proportion of cells in sub-G1 phases. (E) Statistical analysis of the proportion of cells in early and late apoptosis. (F) Representative images of the flow cytometry of PANC-1 cells treated with shCon, shPPP5C or combined treatment of GEM and shCon or shPPP5C. Data are presented as the mean \pm standard deviation of 3 independent experiments. ${ }^{*} \mathrm{P}<0.05,{ }^{* *} \mathrm{P}<0.01,{ }^{* * *} \mathrm{P}<0.001 . \mathrm{GEM}$, gemcitabine; shPPP5C, short hairpin serine/threonine protein phosphatase 5; shCon, short hairpin control.

shPPP5C resulted in a marked increase in p-JNK/JNK, cleaved caspase 3 and PARP, while the expression ratio of p-p38/p38 and p-p53/p53 exhibited almost no change, suggesting that PPP5C silencing combined with GEM may, to a certain extent, promote further cell apoptosis (Fig. 4C).

\section{Discussion}

The majority of PC patients exhibit a strong resistance to chemotherapy. The exact mechanisms underlying this remain unknown. One underlying mechanism of chemoresistance 
$\mathbf{A}$

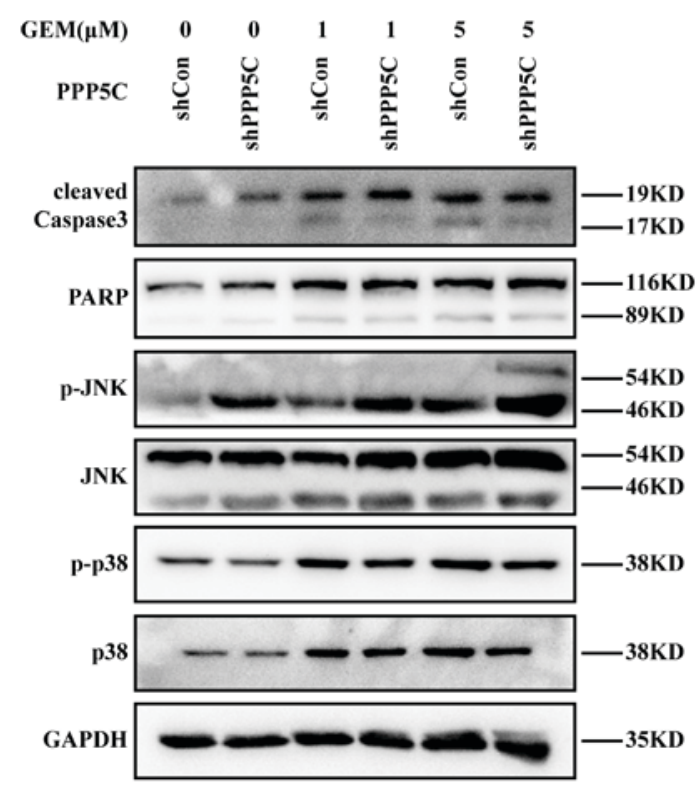

C

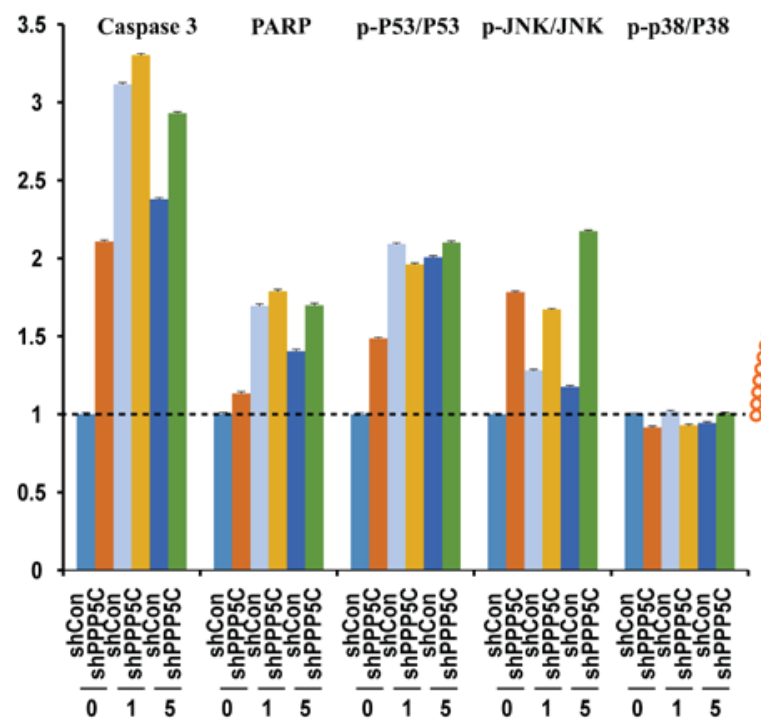

B

\begin{tabular}{|c|c|c|c|c|c|}
\hline $\operatorname{GEM}(\mu \mathrm{M})$ & 0 & 1 & 5 & 0 & 1 \\
\hline PPP5C & 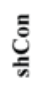 & 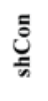 & ప్ర్ & 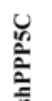 & U. \\
\hline
\end{tabular}
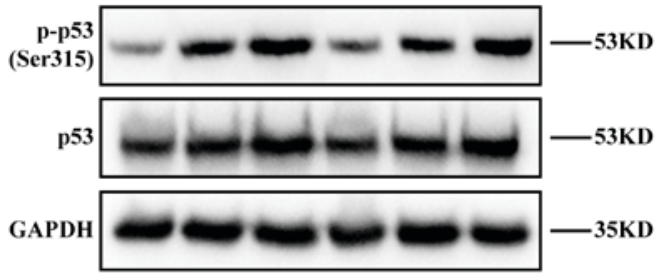

D
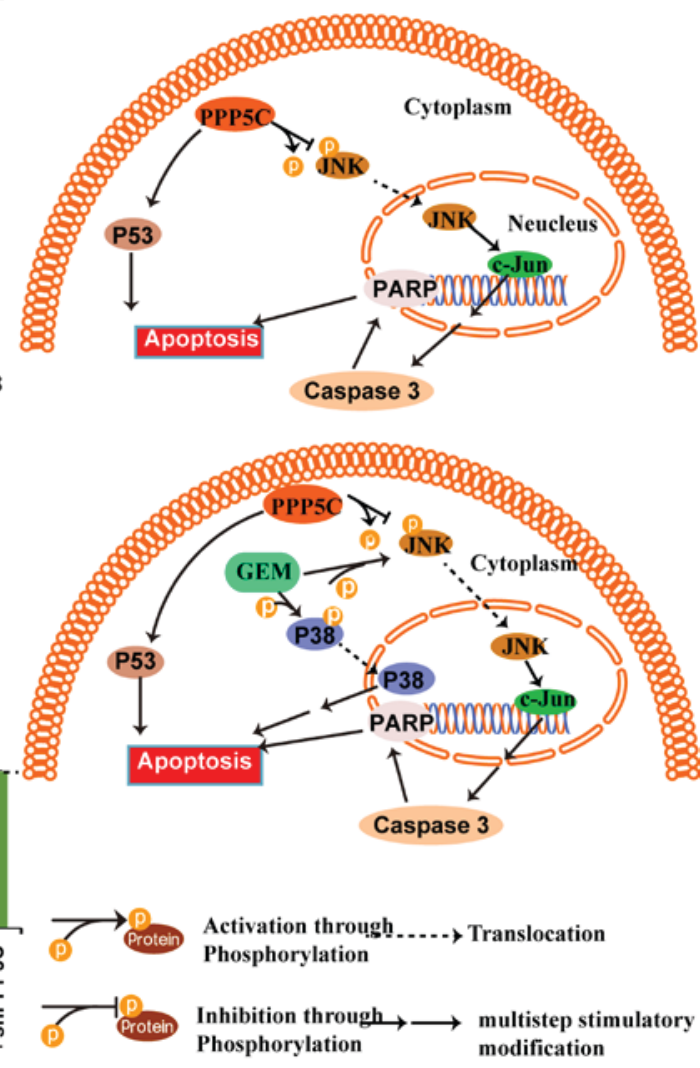

Figure 4. Effects of PPP5C-knockdown on apoptosis pathway-associated molecular expression. (A) The levels of caspase-3, PARP, JNK, p-JNK, p38 and p-p38 protein in PANC-1 cells were detected following PPP5C silencing by western blot analysis. (B) The levels of p-p53 (ser315) and p53 protein in PANC-1 cells were analyzed following PPP5C silencing by western blot analysis. (C) The expression of JNK, p-JNK, p38 and p-p38 proteins in PANC-1 cells transduced with shCon, shPPP5C or the combined treatment of GEM and shPPP5C or shCon was analyzed by western blot analysis. (D) Proposed model of the role served by PPP5C silencing in cell apoptosis. GAPDH was used as an internal loading control. PPP5C, serine/threonine protein phosphatase 5; JNK, c-Jun N-terminal kinases; shCon, short hairpin control; shPPP5C, short hairpin PPP5C; GEM, gemcitabine; p-, phosphorylated-; t-, total-.

is the insensitivity to drug-induced apoptosis (38). GEM is widely used for several cancer types, not only for PC, but also for lung and bladder cancer types. GEM monotherapy has been the standard treatment for metastatic PC for decades and combination therapy of GEM with a number of other agents has been demonstrated to increase the median survival time to a certain extent (9). However, although GEM is still used as a first-line treatment option, the modest survival benefit of GEM treatment has been revealed to be unsatisfactory (39). GEM is a unique antimetabolite that may inhibit the activity of ribonucleotide reductase and may terminate DNA elongation processes (40). However, the occurrence of drug resistance is common in cancer treatment with various agents due to multiple factors, including the attenuation of nucleoside transporters, the augmentation of efflux transporters, the acquisition of apoptotic resistance due to overexpression of anti-apoptotic proteins (e.g., heat shock proteins, cyclooxygenase-2, nuclear factor erythroid 2-related factor 2 and B-cell lymphoma 2 family members), and the constitutive activation of survival signaling pathways $(16,41)$. Therefore, GEM resistance is a significant obstacle to effective chemotherapy.

It has been reported that the overexpression of PPP5C has been observed in several solid cancer types (42-44). In addition, overexpression of PPP5C has been demonstrated to promote cell proliferation and tumor progression (35). PPP5C expression is responsive to hypoxia inducible factor-1 and estrogen, 
which plays important role in cancer progression. It has been reported that PPP5C serves an important role in glioma metastasis, as downregulation of PPP5C mitigated cell migration in U251 and U373 cell lines (34). In human breast cancer, a strong association was observed between high levels of PPP5C expression and the occurrence of invasive ductal carcinoma in patients with metastatic disease at the time of diagnosis (45). In rats, PPP5C mRNA levels markedly increased in malignant ascites hepatomas (46). Furthermore, a protein microarray analysis of mantle-cell lymphoma also revealed elevated PPP5C expression (47). With regards to PC, increased PPP5C expression was observed in PANC-1 cells treated with GEM in the present study. The association between PPP5C levels and tumor progression requires elucidation in order to find an effective strategy to overcome GEM resistance.

Based upon previous reports of PPP5C in other cancer types and the observation of PPP5C overexpression in PANC-1 cells by GEM treatment, we hypothesized that the suppression of PPP5C expression may serve an important role in PC growth and may promote the effect of GEM treatment on PC cells. In the present study, the effect of PPP5C-knockdown or the combination of this with GEM treatment on the cellular functions of PANC-1 cell was investigated. The results of the MTT assay demonstrated that the proliferation of PANC-1 cells was significantly impaired in the combination treatment group, which indicated that PPP5C silencing enhanced the chemosensitivity of PANC-1 cells to GEM. Additionally, the effects of PPP5C-knockdown alone or the combination treatment of GEM and PPP5C silencing on cell cycle and apoptosis were also studied. The results demonstrated that suppressed PPP5C expression combined with GEM treatment in PANC-1 cells led to cell arrest in the $G_{0} / G_{1}$ phase and increased cell apoptosis. Therefore, these results suggest that PPP5C may serve a central role in the tumor progression process of PC and in GEM resistance.

In particular, PPP5 has been revealed to act as a suppressor of ASK $(48,49)$, p53 and DNA-dependent protein kinase, catalytic subunit (45). PPP5C appears to interact with ASK1, which is associated with the c-JNK and p38 signaling pathways. The present study observed that, in the shPPP5C silencing group, the protein expression ratio of $\mathrm{p}-\mathrm{JNK} / \mathrm{JNK}$ was increased, while almost no change was observed in that of p-P38/P38. Meanwhile, combined treatment with GEM and shPPP5C also resulted in a marked increase in $\mathrm{p}-\mathrm{JNK} / \mathrm{JNK}$ and no change in p-p38/p38. These results indicate that the combined treatment could further induce cell apoptosis via the c-JNK pathway. Furthermore, western blot analysis demonstrated that PPP5C silencing significantly increased the protein levels of cleaved caspase-3, p-p53 and cleaved-PARP, which participate in the cell apoptotic pathway. As the prime activator of PARP is DNA damage and its overexpression has been proven to be associated with the pathogenesis of numerous tumors (50), there may be several links between p53 and PARP. These findings support the hypothesis that the involvement of MAPK signaling pathways affects the results of anticancer therapy on PC cells (51). Combined with the previous findings that the activation of the p38 kinase promotes the activation of p53 and further triggers the activation of caspase-9 and -3 in PC (52), the results of the present study revealed that the downstream target of PPP5C is p53 (Fig. 4D). It is widely acknowledged that MAPKs are responsive to various stress stimuli. Upon activation, JNK phosphorylates and regulates various cell cycle and apoptotic mediators. p-p53 may initiate the p53 response and may lead to cell arrest, as well as apoptosis within cell cycles (53). However, the detailed mechanisms underlying this remain to be elucidated.

In summary, the present study demonstrated that PPP5C silencing has a potential novel therapeutic function in $\mathrm{PC}$. Further investigation is required to elucidate the precise mechanisms of PPP5C for PC treatment with GEM, such that a novel gene-targeted therapy for PC may be established.

\section{Acknowledgements}

The authors would like to thank the National Natural Science Foundation of China (grant no. 81201733), the Zhejiang Provincial Medicine Foundation (grant no. 2012KYB018) and the Zhejiang Provincial Chinese Medicine Program (grant no. 2013ZQ021) for providing financial support.

\section{Competing interests}

The authors declare that they have no competing interests.

\section{References}

1. Wolfgang CL, Herman JM, Laheru DA, Klein AP, Erdek MA Fishman EK and Hruban RH: Recent progress in pancreatic cancer. CA Cancer J Clin 63: 318-348, 2013.

2. Hurton S, MacDonald F, Porter G, Walsh M and Molinari M: The current state of pancreatic cancer in Canada: Incidence, mortality, and surgical therapy. Pancreas 43: 879-885, 2014.

3. Miwa T, Kokuryo T, Yokoyama Y, Yamaguchi J and Nagino M: Therapeutic potential of targeting protein for Xklp2 silencing for pancreatic cancer. Cancer Med 4: 1091-1100, 2015.

4. Zuo J, Zhang C, Ren C, Pang D, Li Y, Xie X, Tang Z and Jiang X: Secretory leukocyte protease inhibitor is a proliferation and survival factor for pancreatic cancer cells. Clin Transl Oncol 17: 314-321, 2015.

5. Siegel R, Naishadham D and Jemal A: Cancer statistics, 2013. CA Cancer J Clin 63: 11-30, 2013.

6. Funel N, Del Chiaro M, Cahen DL and Laukkarinen J: Pancreatic cancer. Gastroenterol Res Pract 2015: 809036, 2015.

7. Thota R, Pauff JM and Berlin JD: Treatment of metastatic pancreatic adenocarcinoma: A review. Oncology (Williston Park) 28: 70-74, 2014.

8. Oettle H, Post S, Neuhaus P, Gellert K, Langrehr J, Ridwelski K, Schramm H, Fahlke J, Zuelke C, Burkart C, et al: Adjuvant chemotherapy with gemcitabine vs observation in patients undergoing curative-intent resection of pancreatic cancer: A randomized controlled trial. JAMA 297: 267-277, 2007.

9. Burris HA III, Moore MJ, Andersen J, Green MR, Rothenberg ML, Modiano MR, Cripps MC, Portenoy RK, Storniolo AM, Tarassoff P, et al: Improvements in survival and clinical benefit with gemcitabine as first-line therapy for patients with advanced pancreas cancer: A randomized trial. J Clin Oncol 15: 2403-2413, 1997.

10. Tempero MA, Behrman S, Ben-Josef E, Benson AB III, Cameron JL, Casper ES, Hoffman JP, Karl RC, Kim P, Koh WJ, et al: Pancreatic adenocarcinoma: Clinical Practice Guidelines in Oncology. J Natl Compr Canc Netw 3: 598-626, 2005.

11. Oettle H and Riess H: Gemcitabine in combination with 5-fluorouracil with or without folinic acid in the treatment of pancreatic cancer. Cancer 95: 912-922, 2002.

12. Poplin E, Feng Y, Berlin J, Rothenberg ML, Hochster H, Mitchell E, Alberts S, O'Dwyer P, Haller D, Catalano P, et al: Phase III, randomized study of gemcitabine and oxaliplatin versus gemcitabine (fixed-dose rate infusion) compared with gemcitabine (30-minute infusion) in patients with pancreatic carcinoma E6201: A trial of the Eastern Cooperative Oncology Group. J Clin Oncol 27: 3778-3785, 2009. 
13. Colucci G, Labianca R, Di Costanzo F, Gebbia V, Cartenì G, Massidda B, Dapretto E, Manzione L, Piazza E, Sannicolò M, et al: Randomized phase III trial of gemcitabine plus cisplatin compared with single-agent gemcitabine as first-line treatment of patients with advanced pancreatic cancer: The GIP-1 study. J Clin Oncol 28: 1645-1651, 2010.

14. Chauffert B, Mornex F, Bonnetain F, Rougier P, Mariette C, Bouché O, Bosset JF, Aparicio T, Mineur L, Azzedine A, et al: Phase III trial comparing intensive induction chemoradiotherapy (60 Gy, infusional 5-FU and intermittent cisplatin) followed by maintenance gemcitabine with gemcitabine alone for locally advanced unresectable pancreatic cancer. Definitive results of the 2000-01 FFCD/SFRO study. Ann Oncol 19: 1592-1599, 2008.

15. Philip PA, Benedetti J, Corless CL, Wong R, O'Reilly EM, Flynn PJ, Rowland KM, Atkins JN, Mirtsching BC, Rivkin SE, et al: Phase III study comparing gemcitabine plus cetuximab versus gemcitabine in patients with advanced pancreatic adenocarcinoma: Southwest Oncology Group-directed intergroup trial S0205. J Clin Oncol 28: 3605-3610, 2010.

16. Minami K, Shinsato Y, Yamamoto M, Takahashi H, Zhang S, Nishizawa Y, Tabata S, Ikeda R, Kawahara K, Tsujikawa K, et al: Ribonucleotide reductase is an effective target to overcome gemcitabine resistance in gemcitabine-resistant pancreatic cancer cells with dual resistant factors. J Pharmacol Sci 127 319-325, 2015

17. Cohen PT: Novel protein serine/threonine phosphatases: Variety is the spice of life. Trends Biochem Sci 22: 245-251, 1997.

18. Cohen PT, Brewis ND, Hughes V and Mann DJ: Protein serine/threonine phosphatases; an expanding family. FEBS Lett 268: 355-359, 1990

19. Morita K, Saitoh M, Tobiume K, Matsuura H, Enomoto S, Nishitoh H and Ichijo H: Negative feedback regulation of ASK1 by protein phosphatase 5 (PP5) in response to oxidative stress. EMBO J 20: 6028-6036, 2001

20. Douglas P, Moorhead GB, Ye R and Lees-Miller SP: Protein phosphatases regulate DNA-dependent protein kinase activity. J Biol Chem 276: 18992-18998, 2001.

21. von Kriegsheim A, Pitt A, Grindlay GJ, Kolch W and Dhillon AS: Regulation of the Raf-MEK-ERK pathway by protein phosphatase 5. Nat Cell Biol 8: 1011-1016, 2006.

22. Yong WH, Ueki K, Chou D, Reeves SA, von Deimling A, Gusella JF, Mohrenweiser HW, Buckler AJ and Louis DN: Cloning of a highly conserved human protein serine-threonine phosphatase gene from the glioma candidate region on chromosome 19q13.3. Genomics 29: 533-536, 1995.

23. Yang J, Roe SM, Cliff MJ, Williams MA, Ladbury JE, Cohen PT and Barford D: Molecular basis for TPR domain-mediated regulation of protein phosphatase 5. EMBO J 24: 1-10, 2005.

24. Hinds TD Jr. and Sánchez ER: Protein phosphatase 5. Int J Biochem Cell Biol 40: 2358-2362, 2008.

25. Chinkers M: Protein phosphatase 5 in signal transduction. Trends Endocrinol Metab 12: 28-32, 2001.

26. Golden T, Swingle M and Honkanen RE: The role of serine/threonine protein phosphatase type 5 (PP5) in the regulation of stress-induced signaling networks and cancer. Cancer Metastasis Rev 27: 169-178, 2008.

27. Swingle MR, Honkanen RE and Ciszak EM: Structural basis for the catalytic activity of human serine/threonine protein phosphatase-5. J Biol Chem 279: 33992-33999, 2004.

28. Zuo Z, Dean NM and Honkanen RE: Serine/threonine protein phosphatase type 5 acts upstream of $\mathrm{p} 53$ to regulate the induction of p21(WAF1/Cip1) and mediate growth arrest. J Biol Chem 273: $12250-12258,1998$

29. Zhao S and Sancar A: Human blue-light photoreceptor hCRY2 specifically interacts with protein serine/threonine phosphatase 5 and modulates its activity. Photochem Photobiol 66: 727-731, 1997.

30. Yamaguchi Y, Katoh H, Mori K and Negishi M: Galpha(12) and Galpha(13) interact with Ser/Thr protein phosphatase type 5 and stimulate its phosphatase activity. Curr Biol 12: 1353-1358, 2002

31. Chatterjee A, Wang L, Armstrong DL and Rossie S: Activated Rac1 GTPase translocates protein phosphatase 5 to the cell membrane and stimulates phosphatase activity in vitro. J Biol Chem 285: 3872-3882, 2010.

32. Hattori K, Naguro I, Runchel C and Ichijo H: The roles of ASK family proteins in stress responses and diseases. Cell Commun Signal 7: 9, 2009.
33. Zheng X, Zhang L, Jin B, Zhang F, Zhang D and Cui L: Knockdown of protein phosphatase 5 inhibits ovarian cancer growth in vitro. Oncol Lett 11: 168-172, 2016.

34. Zhi X, Zhang H, He C, Wei Y, Bian L and Li G: Serine/threonine protein phosphatase-5 accelerates cell growth and migration in human glioma. Cell Mol Neurobiol 35: 669-677, 2015.

35. Feng L, Sun P, Li Z, Liu M and Sun S: Knockdown of PPP5C inhibits growth of hepatocellular carcinoma cells in vitro. Appl Biochem Biotechnol 175: 526-534, 2015.

36. Liu F, Iqbal K, Grundke-Iqbal I, Rossie S and Gong CX Dephosphorylation of tau by protein phosphatase 5: Impairment in Alzheimer's disease. J Biol Chem 280: 1790-1796, 2005.

37. Livak KJ and Schmittgen TD: Analysis of relative gene expression data using real-time quantitative PCR and the 2-(delta delta C(T)) method. Methods 25: 402-408, 2001

38. Gottesman MM: Mechanisms of cancer drug resistance. Annu Rev Med 53: 615-627, 2002.

39. Moore MJ, Goldstein D, Hamm J, Figer A, Hecht JR, Gallinger S, Au HJ, Murawa P, Walde D, Wolff RA, et al: Erlotinib plus gemcitabine compared with gemcitabine alone in patients with advanced pancreatic cancer: A phase III trial of the National Cancer Institute of Canada Clinical Trials Group. J Clin Oncol 25: 1960-1966, 2007.

40. Huang P, Chubb S, Hertel LW, Grindey GB and Plunkett W: Action of 2',2'-difluorodeoxycytidine on DNA synthesis. Cancer Res 51: 6110-6117, 1991.

41. Li L and Leung PS: Use of herbal medicines and natural products: An alternative approach to overcoming the apoptotic resistance of pancreatic cancer. Int J Biochem Cell Biol 53: 224-236, 2014

42. Golden T, Aragon IV, Zhou G, Cooper SR, Dean NM and Honkanen RE: Constitutive over expression of serine/threonine protein phosphatase 5 (PP5) augments estrogen-dependent tumor growth in mice. Cancer Lett 215: 95-100, 2004.

43. Amable L, Grankvist N, Largen JW, Ortsäter H, Sjöholm ^ and Honkanen RE: Disruption of serine/threonine protein phosphatase 5 (PP5:PPP5c) in mice reveals a novel role for PP5 in the regulation of ultraviolet light-induced phosphorylation of serine/threonine protein kinase Chk1 (CHEK1). J Biol Chem 286: 40413-40422, 2011

44. Jeong JY, Johns J, Sinclair C, Park JM and Rossie S: Characterization of Saccharomyces cerevisiae protein Ser/Thr phosphatase T1 and comparison to its mammalian homolog PP5. BMC Cell Biol 4: 3, 2003

45. Golden T, Aragon IV, Rutland B, Tucker JA, Shevde LA, Samant RS, Zhou G, Amable L, Skarra D and Honkanen RE: Elevated levels of Ser/Thr protein phosphatase 5 (PP5) in human breast cancer. Biochim Biophys Acta 1782: 259-270, 2008.

46. Shirato H, Shima H, Nakagama H, Fukuda H, Watanabe Y, Ogawa K, Matsuda Y and Kikuchi K: Expression in hepatomas and chromosomal localization of rat protein phosphatase 5 gene. Int J Oncol 17: 909-912, 2000.

47. Ghobrial IM, McCormick DJ, Kaufmann SH, Leontovich AA, Loegering DA, Dai NT, Krajnik KL, Stenson MJ, Melhem MF, Novak AJ, et al: Proteomic analysis of mantle-cell lymphoma by protein microarray. Blood 105: 3722-3730, 2005.

48. Zhou G, Golden T, Aragon IV and Honkanen RE: Ser/Thr protein phosphatase 5 inactivates hypoxia-induced activation of an apoptosis signal-regulating kinase $1 / \mathrm{MKK}-4 / \mathrm{JNK}$ signaling cascade. J Biol Chem 279: 46595-46605, 2004

49. Huang S, Shu L, Dilling MB, Easton J, Harwood FC, Ichijo H and Houghton PJ: Sustained activation of the JNK cascade and rapamycin-induced apoptosis are suppressed by p53/p21(Cip1). Mol Cell 11: 1491-1501, 2003

50. Michels J, Obrist F, Castedo M, Vitale I and Kroemer G: PARP and other prospective targets for poisoning cancer cell metabolism. Biochem Pharmacol 92: 164-171, 2014.

51. Hong MY, Gao JL, Cui JZ, Wang KJ, Tian YX, Li R, Wang HT and Wang $\mathrm{H}$ : Effect of c-Jun $\mathrm{NH}_{2}$-terminal kinase-mediated p53 expression on neuron autophagy following traumatic brain injury in rats. Chin Med J (Engl) 125: 2019-2024, 2012

52. Bu HQ, Liu DL, Wei WT, Chen L, Huang H, Li Y and Cui JH: Oridonin induces apoptosis in SW1990 pancreatic cancer cells via p53- and caspase-dependent induction of p38 MAPK. Oncol Rep 31: 975-982, 2014

53. Harris SL and Levine AJ: The p53 pathway: Positive and negative feedback loops. Oncogene 24: 2899-2908, 2005. 